\title{
Rapid cell search in OFDM-based cellular systems
}

\author{
Jin-Woo Lee and Yong-Hwan Lee \\ School of Electrical Engineering and INMC, Seoul National University, Kwanak P. O. Box 34, Seoul, 151-600 Korea \\ e-mail: ylee@snu.ac.kr
}

\begin{abstract}
Cell search is one of the most important issues in cellular systems. There have been large efforts on the synchronization problem in OFDM systems. However, conventional OFDM-based cellular schemes may have problems in initial timing acquisition and cell identification, requiring a large cell search time. In this paper, we propose a novel three-step cell search scheme using a new preamble for OFDM-based cellular systems. Simulation results show that the proposed cell search scheme can provide fast cell search performance in multi-cell environment, while reducing the computational complexity.
\end{abstract}

\section{INTRODUCTION}

Orthogonal frequency division multiplexing (OFDM) has been considered as one of the most promising transmission techniques for wideband wireless systems [1, 2]. Recently, it has attracted much attention for the next generation (called 4G) wireless access system. When it is applied to the cellular system, it may need a rapid cell search scheme in mobile channel environment. The cell search process includes the acquisition of initial symbol/frame timing and identification of cell.

There have been a number of researches on the acquisition of initial timing in OFDM systems. However, few researches consider the acquisition in multi-cell environment. The use of a cyclic prefix (CP) has widely been used for the timing acquisition due to its simplicity [4]. However, the CP is often corrupted by inter symbol interference (ISI) in multi-path channel environment. Moreover, since the synchronization is often based on the correlation with the $\mathrm{CP}$, which is directly proportional to the transmit power from the base station, the user can be synchronized to an adjacent base station having higher power than the correct base station. As a result, as the number of users in a cell increases, the more likely new users are to be synchronized to that base station, causing severe unbalancing in the cellular network load. The preamble has also been used to obtain accurate symbol timing. By using a preamble having two repetitive patterns, the computational complexity problem can be alleviated in preamble-based methods [7]. However, this method may suffer from high ambiguity of the timing metric at the boundary of symbol timing instant, degrading the synchronization performance. Moreover, it does not consider multi-cell environment.

In multi-cell environment, initial cell search can efficiently be achieved by using a multi-step approach [8]. Although many researches on have been devoted to the CDMA system, but not much to the OFDM system. In this paper, we propose a novel preamble-based cell search scheme in OFDM cellular systems. The proposed scheme can provide rapid cell search by using a three-step approach. Unlike conventional schemes $[5,6]$, the proposed scheme first finds out the frame timing in a computationally efficient manner. By using a new preamble pattern, it can resolve the ambiguity of the timing metric, providing robust performance in multi-path channel environment.

This paper is organized as follows. Section II describes the OFDM system model. In Section III, we propose a novel cell search scheme with the use of a new preamble pattern. The performance of the proposed cell search scheme is analyzed and verified by computer simulation in terms of the mean cell search time. The computational complexity issues are also considered in Section IV. Finally, conclusions are given in Section V.

\section{OFDM SYSTEM MODEL}

The OFDM transmitter converts the frequency domain information symbol $X[k]$ into the time domain signal by inverse fast Fourier transform (IFFT). The last $N_{g}$ samples are inserted as a CP to form the OFDM symbol, $s[n]$, represented as

$$
s[n]=\frac{1}{\sqrt{N_{c}}} \sum_{k=0}^{N_{c}-1} X[k] e^{j 2 \pi\left(k / N_{c}\right) n},-N_{g} \leq n \leq N_{c}-1
$$

where $N_{c}$ denotes the number of subcarriers and $j=\sqrt{-1}$. The signal $s[n]$ is transmitted over a frequency selective multi-path channel whose impulse response is represented as

$$
h[n]=\sum_{l=0}^{L-1} h_{l}[n] \delta\left[n-n_{l}\right]
$$

where $h_{l}$ denotes the complex channel coefficient, $n_{l}$ denotes the time delay of the $l$-th multi-path component, and $L$ denotes 
the number of multi-paths.

The received signal $r[n]$ can be represented as

$$
r[n]=h[n] * s[n]+i[n]
$$

where $i[n]$ represents the noise plus interference term and $*$ denotes the convolution process. If the timing is perfectly synchronized, the received symbol $Y[k]$ is obtained by FFT after discarding the CP of $N_{g}$ samples from $r[n]$, given by

$$
Y[k]=X[k] H[k]+I[k]
$$

where $k$ denotes the subcarrier index, $I[k]$ represents the noise in the frequency domain and $H[k]$ is the frequency response of the channel represented as

$$
H[k]=\sum_{l=0}^{L-1} h_{l}[n] e^{-j 2 \pi\left(k / N_{c}\right) n_{l}}
$$

When the timing is not perfectly acquired, the received signal in (3) can be expressed as

$$
r[n]=\sum_{l=0}^{L-1} h_{l}\left[n+n_{\varepsilon}\right] s\left[n+n_{\varepsilon}-n_{l}\right]+i\left[n+n_{\varepsilon}\right]
$$

where $n_{\varepsilon}$ denotes the amount of timing offset. If $n_{\varepsilon}$ is in the range of $\left[n_{L-1}-N_{g}, 0\right]$, the information symbol can be recovered without the effect of inter carrier interference (ICI) and ISI. If not, the demodulated signal can be represented as [3]

$$
Y[k]=\frac{N_{c}-n_{\varepsilon}}{N_{c}} X[k] e^{j 2 \pi\left(k / N_{c}\right) n_{\varepsilon}}+I_{I S I}[k]+I_{I C I}[k]+I[k]
$$

where $I_{I S I}[k]$ and $I_{I C I}[k]$ represent the interference due to the ISI and ICI, respectively. Note that, in addition to the interference, the received symbol is attenuated in proportion to the timing offset. As a result, the receiver performance seriously deteriorates. Therefore, it is required to acquire accurate timing during the initial cell search process.

\section{PROPOSED CELL SEARCH SCHEME}

We consider a three-step process for the cell search as shown in Fig. 1. The frame timing $\hat{n}_{f}$ is first acquired by performing a block-wise autocorrelation. It detects the preamble position in a frame. Then the symbol timing $\hat{n}_{s}$ is acquired by autocorrelating first half-symbol with second half-symbol. It acquires the start timing of the FFT window within the preamble range. Finally, the target cell is identified by performing crosscorrelation with cellspecific preamble pattern. It detects the cell identification code $\hat{m}_{c}$ of the nearest cell. In each-step, the decision is achieved by comparing the associated metric with a threshold.

For better performance, we design a new preamble pattern as

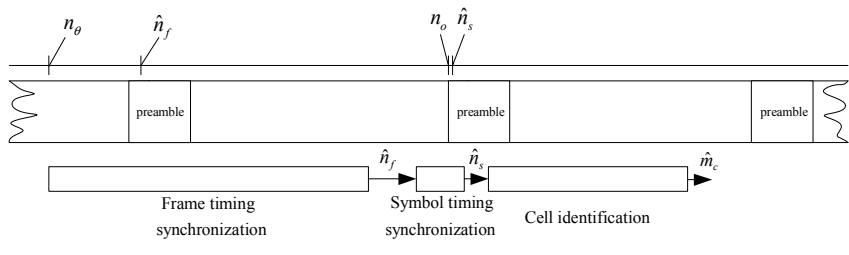

Fig. 1. Proposed cell search process

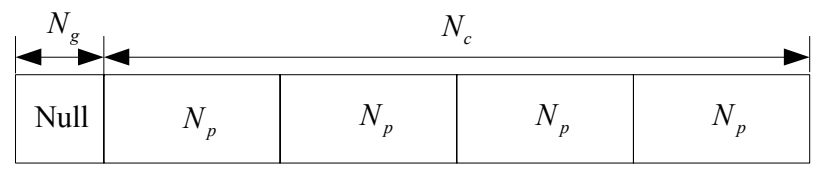

Fig. 2. Structure of the proposed preamble in the time domain

illustrated in Fig. 2. The proposed preamble comprises four repetitive signals of period $N_{p}$ with a guard interval $N_{g}$. This four-time repetitive pattern provides the optimum performance of the block-wise autocorrelation. The proposed preamble does not use any signal in the guard interval to obtain a symbol timing metric with a sharp shape. For the cell identification, each cell uses a preamble pattern among $m_{\text {cell }}$ patterns which are distinguishable from each other. Thus, the preamble signal of the $k$-th subcarrier and the $m$-th cell in the frequency domain can be expressed as

$$
P[k, m]=\left\{\begin{array}{l}
2 q[k / 4, m], k=0,4,8, \cdots, N_{c}-4 \\
0 \quad, \text { otherwise }
\end{array}\right.
$$

where $q[k, m]$ denotes a cell-specific code of length $N_{p}$. We use an extended PN sequence as the cell-specific code that has good autocorrelation and crosscorrelation characteristics. The IFFT of $P[k, m]$ yields a periodic signal with period $N_{p}$ in the time domain as illustrated in Fig. 2. Note that the constant scaling factor 2 in (8) makes both the preamble and OFDM symbol have the same normalized average power.

The frame timing can be detected from the preamble position. Define a timing index set by

$$
\Phi_{f} \triangleq\left\{n: n=n_{\theta}+2 N_{p} \cdot i, i=0,1, \cdots, I_{\theta}\right\}, 0 \leq n_{\theta} \leq N_{f}-1
$$

where $N_{f}$ is the number of samples per frame, $n_{\theta}$ denotes the initial timing offset and $I_{\theta}=\left\lfloor N_{f} /\left(2 N_{p}\right)-1\right\rfloor$. Here, $\lfloor x\rfloor$ denotes the largest integer less than or equal to $x$. The set $\Phi_{f}$ comprises the time instants for the correlation from time $n_{\theta}$. To obtain the preamble position, the receiver performs a block-wise correlation defined as

$$
W_{f}[n]=\sum_{i=0}^{N_{p}-1} r^{*}[n+i] r\left[n+i+N_{p}\right], n \in \Phi_{f}
$$



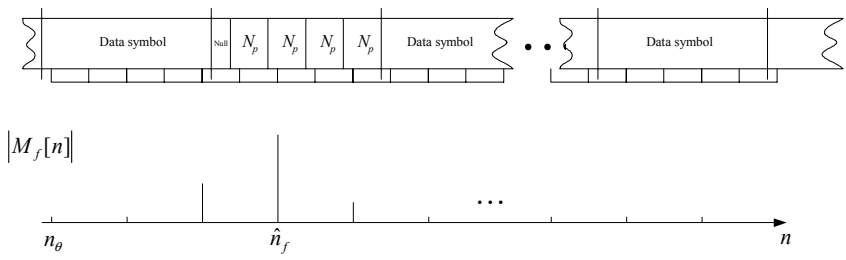

Fig. 3. Frame timing synchronization process

Letting $R_{f}[n]$ be the received energy during the second quarter-symbol

$$
R_{f}[n]=\sum_{i=0}^{N_{p}-1}\left|r\left[n+i+N_{p}\right]\right|^{2}, n \in \Phi_{f}
$$

We have a normalized frame timing metric defined as

$$
M_{f}[n]=\frac{\left|W_{f}[n]\right|^{2}}{R_{f}^{2}[n]}, n \in \Phi_{f}
$$

The frame timing can be found by the timing index $\hat{n}_{f}$ such that

$$
\hat{n}_{f}=\arg \max _{n \in \Phi_{f}}\left|M_{f}[n]\right| \text {, if } \max \left|M_{f}[n]\right| \geq \lambda_{f}
$$

where $\lambda_{f}$ denotes a detection threshold for the frame timing.

The block-wise autocorrelation can significantly reduce the computational complexity of the frame timing acquisition process as shown in Fig. 3. The proposed scheme requires about $N_{p}$ complex multiplications and $N_{p}$ complex additions to proceed $2 N_{p}$ samples. Note that the correlation method in [7] requires one complex multiplication and two complex additions to proceed one sample. Even after completion of initial cell search, the mobile ceaselessly needs to search the frame timing to detect other-cell's preamble for handover. Thus, the proposed scheme can significantly save the power for cell searching for handover.

After finding the frame time $\hat{n}_{f}$, the timing uncertainty is reduced to within a preamble interval. Then, the symbol timing can be found by performing the following autocorrelation

$$
W_{s}[n]=\sum_{i=0}^{2 N_{p}-1} r^{*}[n+i] r\left[n+i+2 N_{p}\right], n \in \Phi_{s}
$$

where $\Phi_{s} \triangleq\left\{\hat{n}_{f}-N_{c}+1, \hat{n}_{f}-N_{c}+2, \cdots, \hat{n}_{f}\right\}$. To reduce the correlation complexity, $W_{s}[n]$ can iteratively be calculated as in [7]. Letting $R_{s}[n]$ be the received energy during the second halfsymbol defined as

$$
R_{s}[n]=\sum_{i=0}^{2 N_{p}-1}\left|r\left[n+i+2 N_{p}\right]\right|^{2}, n \in \Phi_{s}
$$

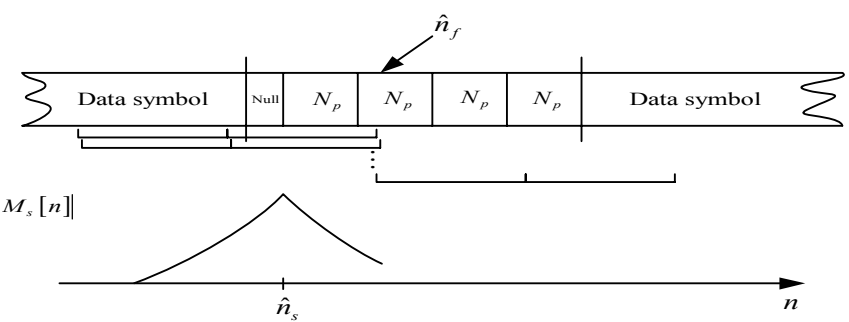

Fig. 4. Symbol timing synchronization process

We can have a normalized symbol timing metric defined as

$$
M_{s}[n]=\frac{\left|W_{s}[n]\right|^{2}}{R_{s}^{2}[n]}, n \in \Phi_{s}
$$

The symbol timing can be found by the timing index $\hat{n}_{s}$ such that

$$
\hat{n}_{s}=\arg \max _{n \in \Phi_{s}}\left|M_{s}[n]\right| \text {, if } \max \left|M_{s}[n]\right| \geq \lambda_{s}
$$

where $\lambda_{s}$ is a detection threshold for the symbol timing.

Since no signal is transmitted during the guard interval, the timing metric has a sharp $\Lambda$ shape as shown in Fig. 4, improving the detection performance. Note that the estimated symbol timing $\hat{n}_{s}$ can be deviated from the correct symbol timing $n_{o}$ by a small amount when the multi-path delay spread is large. If $\hat{n}_{s}-n_{o}=n_{\varepsilon}>0$, the estimated symbol timing should be adjusted to prevent the demodulated signal from corruption by the ISI and ICI as in (7). The sample position of the FFT window $\hat{n}_{w}$ is determined by

$$
\hat{n}_{w}=\hat{n}_{s}-n_{b}
$$

where pre-advanced samples $n_{b}$ should be chosen to be higher than the mean shift of the timing point caused by the channel dispersion. Because $\hat{n}_{w}$ is smaller than $n_{o}$ due to the adjustment, the demodulated symbol can have a phase rotation term as in (7). Thus, the cell identification process should consider this phase rotation effect. To alleviate this phase rotation issue, we detect the cell-specific code using the following differential crosscorrelation.

$$
\begin{aligned}
& W_{c}[m]=\sum_{k=0}^{N_{p}-2}\left(P^{*}[4(k+1), m] R_{\hat{n}_{w}+2 N_{p}}[2(k+1)]\right)\left(P^{*}[4 k, m] R_{\hat{n}_{w}+2 N_{p}}[2 k]\right)^{*}, \\
& m \in \Phi_{c}
\end{aligned}
$$

where $R_{\hat{n}_{w}+2 N_{p}}[k]$ denotes $2 N_{p}$-point FFT at $\left(\hat{n}_{w}+2 N_{p}\right)$ and $\Phi_{c} \triangleq\left\{0,1,2, \cdots, m_{\text {cell }}-1\right\}$. Note that zero power during the guard interval of the preamble may cause an ICI effect in the frequency domain. This problem can be avoided by using $R_{\hat{n}_{w}+2 N_{p}}[k]$ which is free from the delayed signal due to multi-path delay, instead of $R_{\hat{n}_{w}}[k]$. Since the amount of phase rotation is proportional to the 
subcarrier index $k$, the differentially crosscorrelated outputs can be combined coherently. Letting $R_{c}[m]$ be the received signal energy defined as

$$
R_{c}[m]=\sum_{k=0}^{N_{p}-2}\left|R_{\hat{n}_{w}+2 N_{p}}[2 k]\right|^{2}, \quad m \in \Phi_{c}
$$

We can use a normalized cell identification metric defined as

$$
M_{c}[m]=\frac{\left|W_{c}[m]\right|^{2}}{R_{c}^{2}[m]}, m \in \Phi_{c}
$$

Finally, the cell-specific code number can be found by the code index $\hat{m}_{c}$ such that

$$
\hat{m}_{c}=\arg \max _{m \in \Phi_{c}}\left|M_{c}[m]\right|, \text { if } \max \left|M_{c}[m]\right| \geq \lambda_{c}
$$

where $\lambda_{c}$ is a detection threshold for the cell identification. Note that the use of differential crosscorrelation can provide the performance robust to frequency selective fading condition, since the phase differentiated term between the adjacent carriers experiences nearly the same fading.

\section{PERFORMANCE EVALUATION}

The performance of the proposed cell search scheme can be analyzed using a signal flow diagram method. For ease of comparison, the performance is evaluated in terms of the mean cell search time using the Markovian nature of the three-step cell search process $[9,10]$.

The misdetection probability $P_{M_{1}}$ in the first step can be defined as the probability that no frame timing metric exceeds $\lambda_{f}$. In this case, the metric should be recalculated using new samples in the set $\Phi_{f}$ corresponding to the next frame interval (i.e., it will take additional $T_{f}$ seconds). If one or more metric values exceed $\lambda_{f}$, the time corresponding to the largest one is chosen as the framing time. Let $P_{D_{1}}$ and $P_{F_{1}}$ be the probability of correct and false detection of the frame time, respectively. Similarly, we can define the probabilities $P_{D_{2}}, P_{D_{3}}, P_{M_{2}}, P_{M_{3}}, P_{F_{2}}$ and $P_{F_{3}}$, by the probability of correct detection, miss detection and false detection of the symbol time and the cell identification, respectively. If a misdetection occurs in the second or third step, the process goes back to the first step. If the false timing or cell code identification occurs, we assume that the process goes back to the first (i.e., frame timing acquisition) step after a penalty time of $T_{p}$ seconds.

Fig. 5 depicts the signal flow diagram of the proposed cell search process. We can define the branch gain function $H_{1}(z)$, $H_{2}(z), H_{3}(z), H_{4}(z)$ and $H_{5}(z)$ by

$$
H_{1}(z)=\left(1-P_{M_{1}}\right) P_{D_{2}} P_{D_{3}} z^{2 T_{f}}
$$

$$
\begin{gathered}
H_{2}(z)=P_{M_{1}} z^{T_{f}} \\
H_{3}(z)=\left(1-P_{M_{1}}\right)\left\{P_{M_{2}}+\left(1-P_{M_{2}}\right) P_{M_{3}}\right\} z^{2 T_{f}} \\
H_{4}(z)=\left(1-P_{M_{1}}\right)\left\{\left(1-P_{M_{2}}\right) P_{F_{3}}+P_{F_{2}} P_{D_{3}}\right\} z^{2 T_{f}}
\end{gathered}
$$

$$
H_{5}(z)=z^{T_{p}}
$$

Then, it can be shown that the overall generating function is represented as

$$
H(z)=\frac{H_{1}(z)}{1-H_{2}(z)-H_{3}(z)-H_{4}(z) H_{5}(z)}
$$

Thus, the mean cell search time can be obtained by

$$
\begin{aligned}
E\left[T_{\text {search }}\right] & =\left.\frac{d}{d z} H(z)\right|_{z=1} \\
& =\frac{\left(2-P_{M_{1}}\right) T_{f}+\left(1-P_{M_{1}}\right)\left\{\left(1-P_{M_{2}}\right) P_{F_{3}}+P_{F_{2}} P_{D_{3}}\right\} T_{p}}{\left(1-P_{M_{1}}\right) P_{D_{2}} P_{D_{3}}}
\end{aligned}
$$

Based on the analytic results, the performance is evaluated by computer simulation. The simulation parameters are summarized in Table I $[11,12]$. A successful cell search is declaired when the estimated FFT window timing is detected within the guard interval range and correct cell identification code is obtained. We assume that the symbol timing is fine tracked in ISI and ICI free region (i. e., $\left.\left[n_{L-1}-N_{g}, 0\right]\right)$ after the initial acquisition.

Fig. 6 depicts the mean search time of the proposed schemes as a function of the CIR. The thresholds are optimally set to get the best performance in each channel. For fair comparison, we applied the proposed cell identification scheme to the $\mathrm{S} \& \mathrm{C}$ scheme in [7] that does not consider cell identification. It can be seen that the proposed scheme shows significant performance improvement over the S\&C scheme. This is mainly due to that the proposed scheme can efficiently reduce the uncertainty in the first step and sharp shape

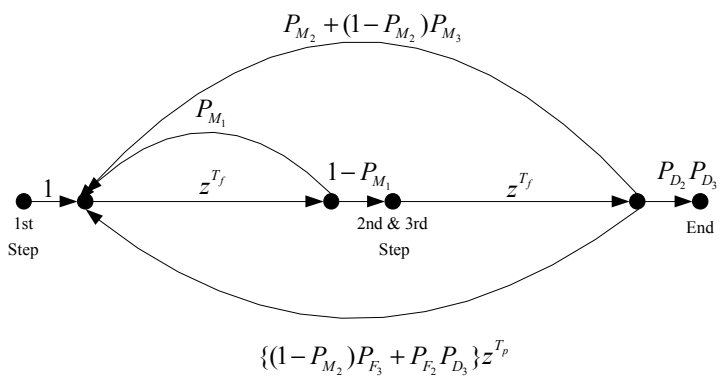

Fig. 5. Signal flow diagram of proposed cell search process 
Table I. Simulation parameters

\begin{tabular}{|l|l|}
\hline Signal bandwidth & $8.75 \mathrm{MHz}$ \\
\hline Number of subcarriers & 1024 \\
\hline Size of FFT and IFFT & 1024 \\
\hline Data modulation & QPSK \\
\hline Frame interval & $5 \mathrm{msec}$ \\
\hline Symbol duration & $102.4 \mu \mathrm{sec}$ \\
\hline Guard interval duration & $12.8 \mu \mathrm{sec}$ \\
\hline False alarm penalty time & $1000 \mathrm{~ms}$ \\
\hline Number of cell codes & 96 \\
\hline Pre-advanced samples & 64 \\
\hline Channel environment & ITU-R M.1225 channel \\
\hline
\end{tabular}

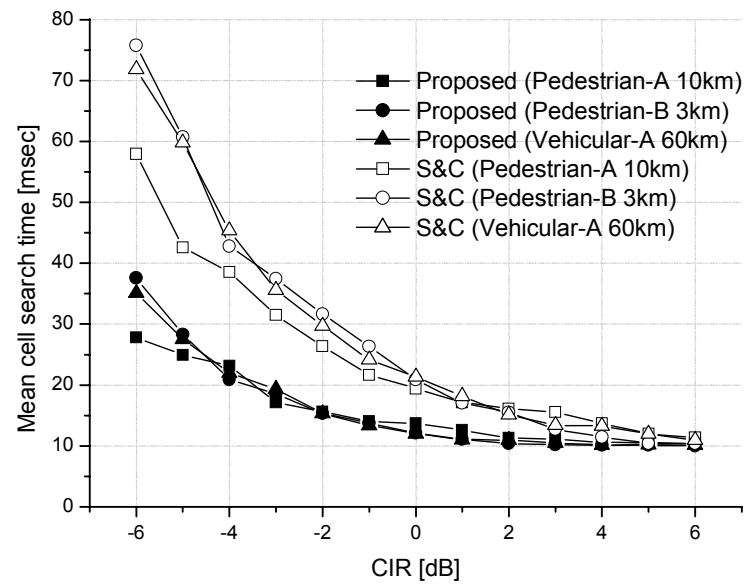

Fig. 6. Mean cell search time

timing metric in the second step with the use of new preamble. Since it has strongest power in dominant paths, cell search performance is best in the pedestrian-A $(10 \mathrm{~km})$ channel at low CIR (e.g., less than $-5 \mathrm{~dB}$ ). However, unless the CIR is too low (e.g., larger than $-4 \mathrm{~dB})$ the performance is best in the pedestrian-B $(3 \mathrm{~km})$ channel due to the frequency diversity effect.

Table II compares the computational complexity in terms of the real addition, multiplication and division of the proposed scheme with the well known $\mathrm{S} \& \mathrm{C}$ scheme in [7]. It can be seen that the proposed scheme requires low computational complexity.

\section{CONCLUSION}

In this paper, we have considered the initial cell search problem in the OFDM-based cellular system. We have proposed a three-step based cell search scheme with the use of a new preamble pattern. The proposed preamble can generate the timing metric with a sharp shape, significantly reducing the uncertainty in finding the frame
Table II. Computational complexity

\begin{tabular}{|c|c|c|c|}
\hline & Addition & Multiplication & Division \\
\hline Proposed & $3 N_{f}$ & $3 N_{f}$ & $2 N_{f} / N_{c}+N_{c}$ \\
\hline S\&C & $10 N_{f}$ & $6 N_{f}$ & $N_{f}$ \\
\hline
\end{tabular}

timing. By applying new processing methods (e.g., block-wise autocorrelation for the frame timing and, differential crosscorrelation for the cell identification), we can significantly reduce the cell search time in addition to the reduction of computational complexity. The performance of the proposed cell search scheme has been analyzed in terms of the mean search time and verified by computer simulation. Simulation results show that the performance improvement over the conventional one is significant when the channel is in bad situation.

\section{REFERENCES}

[1] R. V. Nee and R. Prasad, OFDM for wireless multimedia communications, Altech House, 2000.

[2] J. A. B. Bingham, "Multicarrier modulation for data transmission: An idea whose time has come," IEEE Commun. Mag., vol. 28, pp5-14, May 1990.

[3] M. Speth, S. A. Fechtel, G. Fock, and H. Meyr, "Optimum receiver design for wireless broad-band systems using OFDMpart I," IEEE Trans. Commun., vol. 47, pp. 1668-1677, Nov. 1999.

[4] J. J. van de Beek, M. Sandell, M. Isaksson and P. O. Borjesson, "Low-complex frame synchronization in OFDM systems," in Proc. ICUPC, pp. 982-986, Nov. 1995.

[5] Y. Hanada, K. Higuchi and M. Sawahashi, "Three-step cell search algorithm for broadband multi-carrier CDMA packet wireless access," IEEE Int. Symp., vol. 2, pp. 32-37, Sept. 2001.

[6] M. Tanno, H. Atarashi, K. Higuchi, and M. Sawahashi, "Threestep cell search algorithm exploiting common pilot channel for OFCDM broadband wireless access," IEICE Trans. Commun., vol. E86-B, pp. 325-334, Jan. 2003.

[7] T. M. Schmidl and D. C. Cox, "Robust frequency and timing synchronization for OFDM," IEEE Trans. Commun., vol. 45, pp. 1613-1621, Dec. 1997.

[8] Y.-P.E. Wang and T. Ottosson, "Cell search in W-CDMA," IEEE J. Select. Areas Commun., vol. 18, pp. 1470-1482, Aug. 2000.

[9] A. Polydoros and C. Weber, "A unified approach to serial search spread spectrum acquisition-Part I: General theory," IEEE Trans. Commun., vol. 32, pp. 542-549, May 1984.

[10] E. A. Sourour and S. C. Gupta, "Direct-sequence spreadspectrum parallel acquisition in a fading mobile channel," IEEE Trans. Commun., vol. 38, pp 992-998, July 1990.

[11] TTA_PG302, Specifications for $2.3 \mathrm{GHz}$ band portable internet service, June 2004.

[12] ITU-R, "Guidelines for evaluation of radio transmission technologies for IMT-2000," Recommendation ITU-R M.1225, 1997. 